\title{
On Imbedding Theorems for Besov Spaces of Functions Defined in General Regions
}

\author{
By \\ Tosinobu Muramatu
}

\section{$\S 1$. Introduction and Main Results}

This paper is a continuation of the author's work [7] concerning Besov spaces of functions defined in general regions; here we treat imbedding theorems. Our method is the same as that of the author's previous papers [5] and [7]. That is, we employ an integral representation which gives us Sobolev type inequalities with the aid of the theory of mean interpolation spaces due to Lions-Peetre [3]. To prove the basic inequalities we shall make use of the idea due to O'Neil [10] and Peetre [11].

We denote by $\Omega$ an open set in $n$-space $\mathbf{R}^{n}$. Let $1 \leqq p, \xi \leqq \infty$, and let $s$ be a positive integer such that $s \leqq n$. For measurable functions defined in $\Omega$ we introduce the norm

$$
\|f\|_{L^{p ; n-s(\Omega)}}=\text { ess. } \sup _{x^{\prime \prime} \in \Omega^{\prime \prime}}\left[\int_{\Omega^{\prime}\left(x^{\prime \prime}\right)}\left|f\left(x^{\prime}, x^{\prime \prime}\right)\right|^{p} d x^{\prime}\right]^{1 / p},
$$

where $x=\left(x^{\prime}, x^{\prime \prime}\right), x^{\prime} \in \mathbb{R}^{s}, x^{\prime \prime} \in \mathbb{R}^{n-s}, \Omega^{\prime}\left(x^{\prime \prime}\right)=\left\{x^{\prime} ;\left(x^{\prime}, x^{\prime \prime}\right) \in \Omega\right\}$ and $\Omega^{\prime \prime}$ is the set of all points $x^{\prime \prime}$ such that $\Omega^{\prime}\left(x^{\prime \prime}\right)$ is not empty. Also we make use of the norm

$$
\|f\|_{L_{*}^{p, n-s}(\Omega)}=\text { ess. } \sup _{x^{\prime \prime} \in \Omega^{\prime \prime}}\left[\int_{\Omega^{\prime}\left(x^{\prime \prime}\right)}\left|f\left(x^{\prime}, x^{\prime \prime}\right)\right|^{p} \frac{d x^{\prime}}{|x|^{s}}\right]^{1 / p},
$$

where $|x|$ denotes the Euclidean norm. For any non-negative integer $m$ and $f \in C^{m}(\Omega)$ we define the semi-norm

$$
|f|_{W_{p ; n-s}^{m}(\Omega)}=\sum_{|\alpha|=m}\left\|D^{\alpha} f\right\|_{L^{p, n-s}(\Omega)}
$$

and the norm

Received January 13, 1971. 


$$
\operatorname{li} f\left\|_{W_{p ; n-s(\Omega)}^{m}}=|f|_{W_{p, n-s}^{m}(\Omega)}+\right\| f \|_{L^{p ; n-s(\Omega)},}
$$

where

$$
D^{\alpha}=D_{1}^{\alpha_{1}} \ldots D_{n}^{\alpha_{n}}, D_{k}=\frac{\partial}{\partial x_{k}}, \quad|\alpha|=\alpha_{1}+\cdots+\alpha_{n}
$$

and $\alpha$ stands for a multi-index $\alpha=\left(\alpha_{1}, \ldots, \alpha_{n}\right)$. For any non-negative integer $m$ any positive integer $j$, any real $\tau$ with $0<\tau<j$ and $f \in C^{m}(\Omega)$ we define the semi-norm

$$
|f|_{\substack{m, \xi+r, j \\ p, \xi ; n-s(\Omega)}}
$$

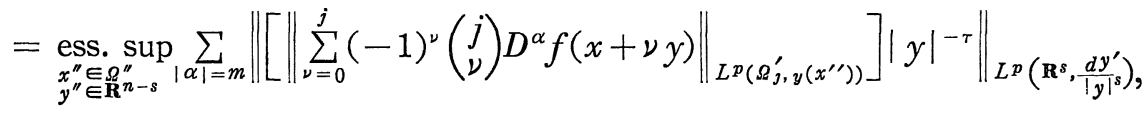

and norm

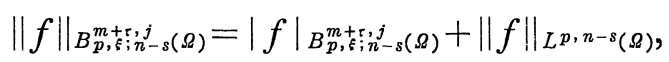

where $\Omega_{j, y}=\bigcap_{\nu=0}^{j}(\Omega-\nu y)$.

Definition 1. The space $W_{p ; n-s}^{m}(\Omega)$ is defined as the completion of the subset of $C^{\infty}(\Omega)$ consisting of functions whose norm given by (1.2) is finite with respect to this norm. Analogously the space $B_{p, \xi ; n-s}^{m+\tau, j}(\Omega)$ is defined as the completion with respect to the norm (1.4).

If $\Omega$ satisfies the cone condition $C\left(T_{0}\right), W_{p ; 0}^{m}(\Omega)=W_{p}^{m}(\Omega)$ coincides with the usual Sobolev space, and $B_{p, \xi ; 0}^{m+\tau, j}(\Omega)$ coincides with the usual Besov space $B_{p, \xi}^{m+\tau}(\Omega)$ (see [7]); while $W_{\infty}^{m}(\Omega)$ coincides with the space $\mathscr{B}^{m}(\Omega)$ of all bounded continuous functions defined in $\Omega$ whose all derivatives of orders $\leqq m$ exist and are continuous and bounded, and $B_{\infty, \infty}^{m+\tau}(\Omega), 0<\tau<1$, is the space $\mathscr{B}^{m+\tau}(\Omega)$ of functions $f \in \mathscr{B}^{m}(\Omega)$ such that for all $|\alpha|=m D^{\alpha} f$ are uniformly Hölder continuous with exponent $\tau$. Here the condition $C\left(T_{0}\right)$ means that is stated in the beginning of $\S 3$. From the results in [7] it is enough to consider the cases where $0<\tau<1=j$ and the case where $\tau=1, j=2$, so that we set for any positive number $\mu$ 
$B_{p, q ; n-s}^{\mu}(\Omega)=\left\{\begin{array}{l}B_{p, q ; n-s}^{m+\tau, 1}(\Omega), \text { if } \mu=m+\tau, 0<\tau<1, m \text { is an integer } \\ B_{p, q ; n-s}^{(\mu-1)+1,2}(\Omega), \text { if } \mu \text { is an integer. }\end{array}\right.$

We state now our main results which are proved in $\S 3$ :

Theorem 1. Assume that $1 \leqq p \leqq q \leqq \infty, 1 \leqq s \leqq n$.

(i) The imbedding operator

$$
W_{p}^{m}(\Omega) \rightarrow W_{q ; n-s}^{k}(\Omega)
$$

exists if $\Omega$ satisfies the condition $C\left(T_{0}\right), m-k-\lambda \geqq 0$, and if either one of the conditions; (a) $m-k-\lambda>0$, or (b) $1<p<q<\infty$, is satisfied, where $\lambda=n / p-s / q$.

(ii) The imbedding operator

$$
W_{p}^{m}(\Omega) \rightarrow B_{q, \eta ; n-s}^{\sigma}(\Omega)
$$

exists if $\Omega$ satisfies the condition $C\left(T_{0}\right), m-\sigma-\lambda \geqq 0$, and if either one of the following conditions is satisfied; (a) $m-\sigma-\lambda>0$, (b) $1<p<q<\infty$, $p \leqq \eta, \quad$ (c) $1<p \leqq \eta \leqq \infty, s<n, \quad$ (d) $\eta=\infty$.

More precisely, under the above conditions the inequalities

$$
\begin{gathered}
|f|_{W_{q ; n-s}^{k}(\Omega)} \leqq C\left\{T^{m-k-\lambda}|f|_{W_{p}^{m}(\Omega)}+T^{-k-\lambda} \|\left. f\right|_{L^{p}(\Omega)}\right\}, \\
|f|_{B_{q, \eta, n-s(\Omega)}^{\sigma} \leqq} \leqq C\left(1+T^{\sigma-r}\right)\left\{T^{m-\sigma-\lambda}|f|_{W_{p}^{m}(\Omega)}+T^{-\sigma-\lambda}\|f\|_{L^{p}(\Omega)}\right\}
\end{gathered}
$$

hold for every $f \in W_{p}^{m}(\Omega)$ and $0<3 T<T_{0}$, where $r$ denotes the greatest integer less than $\sigma$ and $C$ is a constant independent of $f$ and $T$.

Theorem 2. Assume that $1 \leqq p \leqq q \leqq \infty, 1 \leqq s \leqq n$.

(i) The imbedding operator

$$
B_{p, \xi}^{\tau}(\Omega) \rightarrow W_{q ; n-s}^{k}(\Omega)
$$

exists if $\Omega$ satisfies the condition $C\left(T_{0}\right), \tau-k-\lambda \geqq 0$ and if either one 
of the following conditions holds: (a) $\tau>k+\lambda$, (b) $\xi \leqq q<\infty, p<q$, or (c) $\xi=1$; where $\lambda=n / p-s / q$.

(ii) The imbedding operator

$$
B_{p, \xi}^{\tau}(\Omega) \rightarrow B_{q, \eta ; n-s}^{\sigma}(\Omega)
$$

exists if $\Omega$ satisfies the condition $C\left(T_{0}\right), 1 \leqq \xi, \eta \leqq \infty, \tau \geqq \sigma+\lambda$, and if either (a) $\tau>\sigma+\lambda$, or (b) $\xi \leqq \eta$ holds.

That is: under the assumptions stated above the inequalities

$$
\begin{aligned}
& |f|_{W_{q: n-\delta}^{k}(\Omega)} \leqq C\left\{T^{\tau-k-\lambda}|f|_{B_{p, \xi}^{\tau}(\Omega)}+T^{-k-\lambda}\|f\|_{L^{p}(\Omega)}\right\}, \\
& |f|_{B_{q, \eta}^{\sigma} ; n-\delta(\Omega)} \\
& \quad \leqq C\left(1+T^{\sigma-r}\right)\left\{T^{\tau-\sigma-\lambda}|f|_{B_{p, \xi}^{\tau}(\Omega)}+T^{-\sigma-\lambda}\|f\|_{L^{p}(\Omega)}\right\}
\end{aligned}
$$

hold for every $f \in B_{p, \xi}^{r}(\Omega)$ and $0<3 T<T_{0}$, where $r$ denotes the greatest integer less than $\sigma$.

In particular, with the aid of Lemma 2.6 which will be proved in $\S 2$, these theorems yield

Theorem 1'. Let $1 \leqq p \leqq q \leqq \infty, 1 \leqq s<n$, and let $x^{\prime \prime}$ be any fixed point in $\Omega^{\prime \prime}$. Set $\lambda=n / p-s / q$.

(i) Let $\Omega$ be an open set satisfying the condition $C\left(T_{0}\right)$ and assume that (a) $m-k-\lambda>0$, or (b) $m-k-\lambda \geqq 0,1<p<q<\infty$. Then the imbedding operator

$$
W_{p}^{m}(\Omega) \rightarrow W_{p}^{k}\left(\Omega^{\prime}\left(x^{\prime \prime}\right)\right)
$$

exists and its norm is estimated by a constant independent of $x^{\prime \prime}$.

(ii) Let $\Omega$ be an open set satisfying the condition $C\left(T_{0}\right)$, and assume that (a) $m>\sigma+\lambda$, or (b) $m \geqq \sigma+\lambda, 1<p \leqq \eta \leqq \infty$. Then the imbedding operator

$$
W_{p}^{m}(\Omega) \rightarrow B_{q, \eta}^{\sigma}\left(\Omega^{\prime}\left(x^{\prime \prime}\right)\right)
$$

exists and its norm is estimated by a constand independent of $x^{\prime \prime}$. 
Theorem 2'. Let $1 \leqq s<n$, and let $x^{\prime \prime}$ be any fixed point in $\Omega^{\prime \prime}$. Under the assumption stated in Theorem 2, there exist the imbedding operators

$$
B_{p, \xi}^{\tau}(\Omega) \rightarrow W_{q}^{k}\left(\Omega^{\prime}\left(x^{\prime \prime}\right)\right)
$$

and

$$
B_{p, \xi}^{\tau}(\Omega) \rightarrow B_{q, \eta}^{\sigma}\left(\Omega^{\prime}\left(x^{\prime \prime}\right)\right)
$$

with norms not greater than a constant independent of $x^{\prime \prime}$.

The main parts of Theorem 1 and Theorem $1^{\prime}$ have been essentially proved already by Il'in [2]. However his conditions imposed on the region are somewhat complicated, and his proof is too long, in the author's opinion.

Here we shall remark on the norm of Besov spaces. Besov $[1]$ introduced the space $B_{p, \xi}^{\left(\sigma_{1}, \cdots, \sigma_{n}\right)}\left(\mathbf{R}^{n}\right)$ whose norm is given by

$$
\|f\|_{L^{p}\left(\mathbf{R}^{n}\right)}+\sum_{i=1}^{n}\left[\int_{-\infty}^{\infty}\left\|\sum_{\nu=0}^{j_{i}}(-1)^{\nu}\left(\begin{array}{c}
j_{i} \\
\nu
\end{array}\right) D_{i}^{m_{i}} f\left(x+t \nu e_{i}\right)\right\|_{L^{p}\left(\mathbf{R}^{n}\right)} t^{-\tau_{2} \xi-1} d t\right]^{1 / \xi}
$$

where $\sigma_{i}=m_{i}+\tau_{i}, m_{i} \geqq 0, j_{i}>\tau_{i}>0 ; m_{i}, j_{i}$ are integers, and $e_{i}=(0, \ldots, 1$, $\ldots, 0)$. He showed that this space depends only on $\sigma_{1}, \ldots, \sigma_{n} ; p, \xi$, and independent of the choice of $m_{1}, \ldots, m_{n} ; j_{1}, \ldots, j_{n}$. Il'in [2] employed the analogous norm for functions defined in a region $\Omega$ and proved the imbedding theorems. The norm of this kind is dependent on the choice of the coordinate system, furthermore the imbedding theorems are proved for a region more restricted than that discussed in this paper. On the other hand, Taibleson [12] showed that

$$
B_{p, \xi}^{(\sigma, \ldots, \sigma)}\left(\mathbb{R}^{n}\right)=B_{p, \xi}^{\sigma}\left(\mathbb{R}^{n}\right)
$$

where the right hand side is the space defined at the beginning of this section (an operator theoretical proof of this identity is given in [6]). From our investigation it seems that for a general region $\Omega$ the norm given by (1.4) is more natural than that given by (1.9). 
Recently, by means of an operator theoretical method Yoshikawa [14], [15] discussed the imbedding theorems for the case where $\Omega=\mathbf{R}^{n}$.

\section{§2. Interpolation Spaces}

We shall reproduce here some theorems concerning mean interpolation spaces which will be applied later on.

First we shall introduce some notations. Let $(M, \mu)$ be a measure space and let $X$ be a Banach space. By $L^{p}(M, \mu ; X)$ we denote the Banach space of all strongly $\mu$-measurable $X$-valued functions $u(\xi)$ such that

$$
\begin{aligned}
\|u\|_{L^{p}(M, \mu ; X)} & =\left[\int_{M}\|u(\xi)\|_{X}^{p} \mu(d \xi)\right]^{1 / p} & & (1 \leqq p<\infty), \\
& =\underset{\xi}{\text { ess. } \sup \|u(\xi)\|_{X}} & & (p=\infty)
\end{aligned}
$$

is finite. In particular, the space $L^{p}\left(\mathbf{R}^{+}, t^{-1} d t ; X\right)$ is denoted by $L_{*}^{p}\left(\mathbf{R}^{+}\right.$; $X)$, where $\mathbf{R}^{+}$is the set of all positive numbers, and the space $L^{p}(M, \mu ; \mathbb{C})$ is denoted by $L^{p}(M, \mu)$, or $L^{p}(M)$.

Let $X, Y$ be two Banach spaces continuously imbedded in a Hausdorff linear topological space $\mathscr{E}$. Assume that $1 \leqq p, q \leqq \infty,-\infty<\xi, \eta<\infty$ and that $\xi \cdot \eta<0$. Then the mean interpolation spaces $S(p, \xi, X ; q, \eta, Y)$ is the space of elements such that

$$
f=\int_{0}^{\infty} u(t) \frac{d t}{t}
$$

for some function $u(t)$ of $t>0$ with $t^{\xi} u(t) \in L_{*}^{p}\left(\mathbf{R}^{+} ; X\right)$ and $t^{\eta} u(t) \in$ $L_{*}^{q}\left(\mathbf{R}^{+} ; Y\right)$. The norm of $f$ is given by

$$
\inf _{u}\left\{\max \left(\left\|t^{\xi} u(t)\right\|_{L_{*}^{p}\left(\mathbf{R}^{+} ; X\right)},\left\|t^{\eta} u(t)\right\|_{L_{*}^{q}\left(\mathbf{R}^{+} ; Y\right)}\right)\right\},
$$

where $u$ ranges over all functions satisfying the identity (2.2). It is known that

$$
S(p, \lambda \xi, X ; q, \lambda \eta, Y)=S(p, \xi, X ; q, \eta, Y) \quad(\lambda \neq 0)
$$

(cf. Lions-Peetre [3]) with equivalent norms. In the following we shall 
write $S(p, \theta, X ; p, \theta-1, Y)$ by $(X, Y)_{\theta, p}, 0<\theta<1$.

Lemma 2.1. (Peetre $[11]$ ) Let $(M, \mu)$ be a measure space. Assume that $1 \leqq p_{1}, p_{2}, q \leqq \infty, 0<\theta<1$. Then

$$
\left(L^{p_{1}}(M), L^{p_{2}}(M)\right)_{\theta, q}=L^{(p, q)}(M)
$$

with equivalent norms, where

$$
\frac{1}{p}=\frac{1-\theta}{p_{1}}+\frac{\theta}{p_{2}}
$$

and $L^{(p, q)}$ denotes the Lorentz space (cf. [4], [9], [10]).

Lemma 2.2. (Peetre $[11]$ ) Let $(M, \mu)$ be a measure space. Assume that $1 \leqq p, q \leqq \infty$. Then

$$
L^{(p, 1)}(M) \subset L^{(p, q)}(M) \subset L^{(p, \infty)}(M)
$$

with continuous injections. And $L^{(p, p)}=L^{p}$ with equivalent norms.

Combining these lemmas with the reiteration theorem (Lions-Peetre [3]), we obtain

Lemma 2.3. Let $(M, \mu)$ be a measure space and assume that $1 \leqq$ $p_{1}, p_{2}, q_{1}, q_{2}, q \leqq \infty, 0<\theta<1$. Then

$$
\left(L^{\left(p_{1}, q_{1}\right)}(M), L^{\left(p_{2}, q_{2}\right)}(M)\right)_{\theta, q}=L^{(p, q)}(M)
$$

with equivalent norms, where $p$ is given by (2.4).

Lemma 2.4. Let $\Omega$ be an open set in $\mathbf{R}^{n}$, and assume that $1 \leqq p<$ $\infty, 1 \leqq \xi \leqq \infty, 1 \leqq s \leqq n, 0<\theta<1$. Then

$$
\left(L^{p ; n-s}(\Omega), L^{q ; n-s}(\Omega)\right)_{\theta, \xi} \subset L^{(r, \xi) ; n-s}(\Omega),
$$

with continuous injection, where $1 / r=(1-\theta) / p+\theta / q$.

Proof. Let

$$
f(x)=\int_{0}^{\infty} u(t, x) t^{-1} d t,
$$


where $t^{\theta} u(t, x) \in L_{*}^{\xi}\left(\mathbf{R}^{+} ; L^{p ; n-s}(\Omega)\right), t^{\theta-1} u(t, x) \in L_{*}^{\xi}\left(\mathbf{R}^{+} ; L^{q ; n-s}(\Omega)\right)$. Then, for almost every $x^{\prime \prime}$ in $\Omega^{\prime \prime}, t^{\theta} u\left(t, x^{\prime}, x^{\prime \prime}\right) \in L_{*}^{\xi}\left(\mathbf{R}^{+} ; L^{p}\left(\Omega^{\prime}\left(x^{\prime \prime}\right)\right)\right.$ and $t^{\theta-1} u$ $\left(t, x^{\prime}, x^{\prime \prime}\right) \in L_{*}^{\xi}\left(\mathbf{R}^{+} ; L^{q}\left(\Omega^{\prime}\left(x^{\prime \prime}\right)\right)\right.$. Hence, we have

$$
f\left(x^{\prime}, x^{\prime \prime}\right)=\int_{0}^{\infty} u\left(t, x^{\prime}, x^{\prime \prime}\right) t^{-1} d t \in L^{(r, \xi)}\left(\Omega^{\prime}\left(x^{\prime \prime}\right)\right)
$$

and

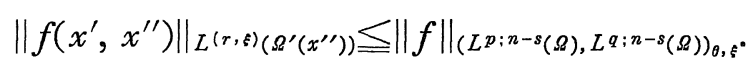

This completes the proof of the lemma.

In the next section we shall frequently make use of the following well known fact:

Lemma 2.5. Let $\left(M_{1}, \mu_{1}\right),\left(M_{2}, \mu_{2}\right)$ be two $\sigma$-finite measure spaces, and let $K(x, y)$ be $a\left(\mu_{1} \times \mu_{2}\right)$-measurable function such that

$$
\begin{array}{ll}
\int_{M_{1}}|K(x, y)|^{r} \mu_{1}(d x) \leqq C_{1}^{r} & \text { for almost all } y \text { in } M_{2}, \\
\int_{M_{2}}|K(x, y)|^{r} \mu_{2}(d y) \leqq C_{2}^{r} & \text { for almost all } x \text { in } M_{1} \\
(1 \leqq r \leqq \infty) . &
\end{array}
$$

Then the integral operator with kernel $K(x, y)$ is a bounded linear operator from $L^{p}\left(M_{2}, \mu_{2}\right)$ into $L^{q}\left(M_{1}, \mu_{1}\right)$ with norm not greater than $C_{1}^{1-r / q}$ $C_{2}^{r / q}$, where $1 / r+1 / p-1 / q=1$. In particular, if

$$
\underset{x, y}{\text { ess. sup }}|K(x, y)| \text {, ess. sup } \int_{y}|K(x, y)| \mu_{1}(d x), \text { ess. } \sup _{x} \int_{M_{2}}|K(x, y)| \mu_{2}(d y)
$$

are finite, then the integral operator with kernel $K(x, y)$ is bounded from $L^{p}\left(M_{2}\right)$ into $L^{q}\left(M_{1}\right)$, where $1 \leqq p \leqq q \leqq \infty$.

Finally we shall prove the following

Lemma 2.6. For every function $f \in B_{p, \xi ; n-s}^{\tau, j}(\Omega)$ and for any fixed point $x^{\prime \prime}$ in $\Omega^{\prime \prime}$

$$
|f|_{B_{p, \xi}^{\mathrm{r}, j}\left(\Omega^{\prime}\left(x^{\prime \prime}\right)\right)} \leqq|f|_{B_{p ; \xi ; n-s}^{\mathrm{r}, j}(\Omega)}
$$

Proof. Set 


$$
F\left(y^{\prime}, y^{\prime \prime}\right)=\int_{\Omega_{j, y}^{\prime}\left(x^{\prime \prime}\right)}\left|\sum_{\nu=0}^{j}(-1)^{\nu}\left(\begin{array}{l}
j \\
\nu
\end{array}\right) f(x+\nu y)\right|^{p} d x^{\prime}
$$

Then by Fatou's lemma we have

$$
F\left(y^{\prime}, 0\right) \leqq \lim _{y^{\prime \prime} \rightarrow 0} F\left(y^{\prime}, y^{\prime \prime}\right)
$$

so that, applying Fatou's lemma again, we obtain

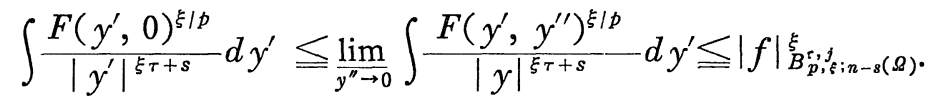

Thus the lemma is proved.

\section{§3. Basic Inequalities}

We say that an open set $\Omega$ satisfies the condition $C\left(T_{0}\right)$ if there exists an $\mathbf{R}^{n}$-valued function $\Psi(x)$ such that (a) $x+t z+t \Psi(x) \in \Omega$ if $x \in \Omega$, $0<t<T_{0},|z| \leqq 1$, and that (b) $\Psi(x)$ is bounded and uniformly continuous in $\mathbf{R}^{n}$. If $\Omega$ satisfies the above condition, then, replacing $T_{0}$ by $T_{0} / \eta$, $\Psi(x)$ by $\eta \Psi * \varphi(x)$, where $\eta>1$ and $\int \varphi(x) d x=1, \varphi \in C_{0}^{\infty}\left(\mathbf{R}^{n}\right)$, the support of $\varphi$ is sufficiently small, we may assume that $\Psi \in \mathscr{B}^{\infty}\left(\mathbf{R}^{n}\right)=\bigcap_{m=1}^{\infty} \mathscr{B}^{m}\left(\mathbf{R}^{n}\right)$. In the following we always assume that $\Omega$ satisfies the condition $C\left(T_{0}\right)$ with $\Psi(x) \in \mathscr{B}^{\infty}\left(\mathbf{R}^{n}\right)$.

In the following of this section we shall discuss some inequalities, which, combining with the integral representations given in [5] and [7], prove the imbedding theorems.

We shall first prove

Lemma 3.1. For $f \in L^{p}(\Omega)$ we define

$$
U_{0}(t, x)=\int_{B}|f(x+t z+t \Psi(x))| d z
$$

Let $1 \leqq p \leqq q \leqq \infty$ and let $1 \leqq \eta \leqq \infty$. Set $\lambda=n / p-s / q$. Then:

(i) For every $f \in L^{p}(\Omega)$ and $0<t<T_{0}$

$$
\left\|U_{0}(t, x)\right\|_{L^{q ; n-s}(\Omega)} \leqq C t^{-\lambda}\|f\|_{L^{p}(\Omega)},
$$


where $C$ is a constant independent of $f$ and $t$,

(ii) For every $f \in L^{p}(\Omega)$ and $0<T<T_{0}$

$$
\left\|\left\{\int_{0}^{T}\left\{U_{0}(t, x) t^{l}\right\}^{\xi} \frac{d t}{t}\right\}^{1 / \xi}\right\|_{L^{q, n-s}(\Omega)} \leqq C T^{l-\lambda}\|f\|_{L^{p}(\Omega)}
$$

if $1 \leqq \xi \leqq q$ and either one of the conditions (a) $l>\lambda$, or (b) $1<p<q<\infty$, $\xi<q, l \geqq \lambda$ is satisfied. Here $C$ is a constant independent of $f$ and $T$.

Also, for every $f \in L^{p}(\Omega)$ and $0<T<T_{0}$

$$
\sup _{x^{\prime \prime} \in \mathbf{R}^{n-s}}\left[\int_{0}^{T}\left\|U_{0}(t, x) t^{l}\right\|_{L^{q\left(\Omega^{\prime}\left(x^{\prime \prime}\right)\right)}} \frac{d t}{t}\right]^{1 / \eta} \leqq C T^{l-\lambda}\|f\|_{L^{p}(\Omega)},
$$

if $l \geqq \lambda$ and if either one of the conditions (a) $l>\lambda$, (b) $1<p<q \leqq \infty$, $p \leqq \eta$, (c) $s<n, 1<p \leqq \eta \leqq \infty$, or (d) $\eta=\infty$ is satisfied.

(iii) For every $f \in L^{p}(\Omega)$ and $0<T<T_{0}$

$$
\begin{gathered}
\sup _{x^{\prime \prime} \in \mathbf{R}^{n-s}}\left\|\left[\left\|\left\{\int_{0}^{T}\left\{U_{0}(t, x) h\left(\frac{|y|}{t}\right)^{k} t^{l}|y|^{-\sigma}\right\}^{\xi} \frac{d t}{t}\right\}^{1 / \xi}\right\|_{L^{q}\left(\Omega^{\prime}\left(x^{\prime \prime}\right)\right)}\right]\right\|_{L_{*}^{\eta ; n-s}\left(\mathbf{R}^{n}\right)} \\
\leqq C T^{l-\sigma-\lambda}\|f\|_{L^{p}(\Omega)}
\end{gathered}
$$

if $1 \leqq \xi \leqq q, \xi \leqq \eta, 0<\sigma<k, l-\sigma-\lambda \geqq 0$, and if either one of the conditions (a) $l-\sigma-\lambda>0$, (b) $1<p<q \leqq \infty, p \leqq \eta$, (c) $1<p \leqq \eta \leqq \infty, s<n$, or (d) $\eta=\infty$ is satisfied. Here $C$ is a constant independent of $f$ and $T$, and $h(t)=\min (t, 1)$.

Proof. (i) Defining $f$ to vanish outside $\Omega$, we have

$$
\left|U_{0}(t, x)\right| \leqq \int_{b B^{\prime \prime}} d z^{\prime \prime} \int_{b B^{\prime}}\left|f\left(x^{\prime}+t z^{\prime}, x^{\prime \prime}+t z^{\prime \prime}\right)\right| d z^{\prime}
$$

where $b=\sup _{x}|\Psi(x)|+1$. Thus for any $x^{\prime \prime}$ in $\mathbb{R}^{n-s}$

$$
\begin{aligned}
& \left\|U_{0}\left(t, x^{\prime}, x^{\prime \prime}\right)\right\|_{L^{q}\left(\Omega^{\prime}\left(x^{\prime \prime}\right)\right)} \\
& \quad \leqq\left(a^{\prime} b^{s}\right)^{(1-1 / p)} \int_{b B^{\prime \prime}}\left\|\left[\int_{b B^{\prime}}|f(x+t z)|^{p} d z^{\prime}\right]^{1 / p}\right\|_{L^{q}\left(\Omega^{\prime}\left(x^{\prime \prime}\right)\right)} d z^{\prime \prime},
\end{aligned}
$$

in virtue of Hö'der's inequality. Combining this with the inequality

$$
\int_{b B^{\prime}}\left|f\left(x^{\prime}+t z^{\prime}, x^{\prime \prime}+t z^{\prime \prime}\right)\right|^{p} d z^{\prime} \leqq t^{-s} g\left(x^{\prime \prime}+t z^{\prime \prime}\right)^{p},
$$


where $g\left(u^{\prime \prime}\right)=\left\|f\left(u^{\prime}, u^{\prime \prime}\right)\right\|_{L^{p}\left(\Omega^{\prime}\left(u^{\prime \prime}\right)\right)}$, we have

$$
\begin{aligned}
& \int\left[\int_{b B^{\prime}}\left|f\left(x^{\prime}+t z^{\prime}, x^{\prime \prime}+t z^{\prime \prime}\right)\right|^{p} d z^{\prime^{\prime}}\right]^{q / p} d x^{\prime} \\
& \leqq\left[g\left(x^{\prime \prime}+t z^{\prime \prime}\right)^{p} t^{-s}\right]^{(q-p) / p} \iint_{b B^{\prime}}\left|f\left(x^{\prime}+t z^{\prime}, x^{\prime \prime}+t z^{\prime \prime}\right)\right|^{p} d x^{\prime} d z^{\prime} \\
& =a^{\prime} b^{s} t^{-s(q-p) / p} g\left(x^{\prime \prime}+t z^{\prime \prime}\right)^{q}
\end{aligned}
$$

which gives

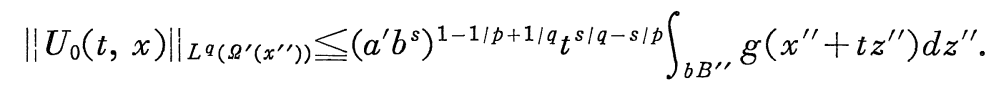

The desired inequality (3.2) now follows from this and Hölder's inequality.

The cases (a) and (d) in (ii) and in (iii) are immediate consequence of (i). Therefore it is sufficient to prove the other cases. We may assume that $l=\lambda$ or $l=\lambda+\sigma$.

We shall prove first (3.4). Consider the case (b). Let $f \in L^{(p, \eta)}(\Omega)$. Then there exist functions $v(t, x)$ and $w(t, x)$ such that

$$
\begin{array}{ll}
f(x)=v(t, x)+w(t, x) & \text { a.e. } t, \\
t^{\kappa \theta} v(t, x) \in L_{*}^{\eta}\left(\mathbb{R}^{+} ; L^{1}(\Omega)\right), t^{\kappa(\theta-1)} w(t, x) \in L_{*}^{\eta}\left(\mathbf{R}^{+} ; L^{q}(\Omega)\right),
\end{array}
$$

where

$$
\theta=\frac{(p-1) q}{(q-1) p} \quad, \quad \kappa=-n+\frac{n}{q} .
$$

Clearly by (3.3) we have

$$
\begin{aligned}
& \left\|\left[\left\|t^{\lambda} U_{0}(t, x)\right\|_{L^{q, n-s}(\Omega)}\right]\right\|_{L_{*}^{\eta}([0, T])} \\
\leqq & C_{1}\left\{\left\|t^{\kappa \theta} v(t, x)\right\|_{L_{*}^{\eta}\left(\mathbf{R}^{+} ; L^{1}(\Omega)\right)}+\left\|_{1}^{l} t^{k(\theta-1)} w(t, x)\right\|_{\left.L_{\left(\mathbf{R}^{+} ; L^{q}(\Omega)\right)}\right\}},\right.
\end{aligned}
$$

which gives

$$
\left\{\int_{0}^{T}\left\|U_{0}(t, x) t^{\lambda}\right\|_{L^{q, n-s}(\Omega)}^{\eta} \frac{d t}{t}\right\}^{1 / \eta} \leqq C\|f\|_{L^{(p, \eta)}(\Omega)}
$$

and (3.4) follows from this, since $L^{p} C L^{(p, \eta)}$ if $p \leqq \eta$. 
Next consider the case (c). It follows from (3.6) that for every $x^{\prime \prime} \in \Omega^{\prime \prime}$,

$$
\begin{aligned}
& \left\|\left[\left\|U_{0}(t, x) t^{l}\right\|_{L^{q}\left(\Omega^{\prime}\left(x^{\prime \prime}\right)\right)}\right]\right\|_{L_{*}^{\eta}\left(\mathbf{R}^{+}\right)} \\
\leqq & C_{1}\left\|t^{(n-s) / p} \int_{b B^{\prime \prime}} g\left(x^{\prime \prime}+t{z^{\prime \prime}}^{\prime \prime}\right) d z^{\prime \prime}\right\|_{L_{*}^{\eta}\left(\mathbf{R}^{+}\right)} \\
= & C_{1}\left\|\int_{b t B^{\prime \prime}} K\left(\left|u^{\prime \prime}\right| / t\right)\left\{\left|u^{\prime \prime}\right|^{(n-s) / p} g\left(x^{\prime \prime}+u^{\prime \prime}\right)\right\}\left|u^{\prime \prime}\right|^{-n+s} d u^{\prime \prime}\right\|_{L_{*}^{\eta}\left(\mathbf{R}^{+}\right)},
\end{aligned}
$$

where $g\left(u^{\prime \prime}\right)=\left\|f\left(u^{\prime}, u^{\prime \prime}\right)\right\|_{L^{p}\left(\Omega^{\prime}\left(u^{\prime \prime}\right)\right)}$ and $K(t)=t^{(n-s)(1-1 / p)}$. Noting that

$$
\begin{aligned}
& \int_{b t B^{\prime \prime}} K\left(\left|u^{\prime \prime}\right| / t\right)\left|u^{\prime \prime}\right|^{-n+s} d u^{\prime \prime}=\frac{a^{\prime \prime} b^{(n-s)(1-1 / p)}}{1-1 / p}, \\
& \int_{\left|u^{\prime \prime}\right| \mid b}^{\infty} K\left(\left|u^{\prime \prime}\right| / t\right) t^{-1} d t=\frac{b^{(n-s)(1-1 / p)}}{(n-s)(1-1 / p)}, \\
& K\left(\left|u^{\prime \prime}\right| / t\right) \leqq b^{(n-s)(1-1 / p)} \text { when } u^{\prime \prime} \in b t B^{\prime \prime},
\end{aligned}
$$

we have (3.4) with the help of Lemma 2.5.

Proof of (3.3). Let $1<p<q<\infty$. Setting $u(t, x)=\left\{t^{\lambda} U_{0}(t, x)\right\}^{\xi}$ for $0<t \leqq T, u(t, x)=0$ otherwise, the left hand side of (3.3) is equal to

$$
\left\|\int_{0}^{\infty} u(t, x) \frac{d t}{t}\right\|_{L^{q \mid \xi ; n-s(\Omega)}}^{\xi}
$$

Take, $\theta, q_{1}, q_{2}$ and $\kappa$ so that

$$
\begin{aligned}
& 1<p<q_{1}<q<q_{2}<\infty, \xi \leqq q_{1}, 0<\theta<1, \\
& \frac{1}{q}=\frac{1-\theta}{q_{1}}+\frac{\theta}{q_{2}}, \quad \kappa=-\frac{s}{q_{1}}+\frac{s}{q_{2}} .
\end{aligned}
$$

Then we have from (3.4)

$$
\begin{aligned}
& \left\|t^{\kappa \theta \xi} u(t, x)\right\|_{L_{*}^{q / \xi}\left(\mathbf{R}^{+} ; L^{q_{1} / \xi ; n-s}(\Omega)\right)}, \\
& \left\|t^{\kappa(\theta-1) \xi} u(t, x)\right\|_{L_{*}^{q / \xi}\left(\mathbf{R}^{+} ; L^{q_{2} / \xi ; n-s}(\Omega)\right)} \\
& \leqq\left\{C\|f\|_{L^{p}(\Omega)}\right\}^{\xi}
\end{aligned}
$$


Since $\left(L^{q_{1} / \xi ; n-s}(\Omega), L^{q_{2} / \xi ; n-s}(\Omega)\right)_{\theta, q / \xi} C L^{q / \xi ; n-s}(\Omega)$, we obtain

$$
\left\|\int_{0}^{\infty} u(t, x) \frac{d t}{t}\right\|_{L^{q \mid \xi ; n-s(\Omega)}} \leqq\left(C\|f\|_{L^{p}(\Omega)}\right)^{\xi}
$$

which is the desired inequalities (3.3).

Proof of part (iii). By Lemma 2.5 we can easily see that the integral operator with the kernel

$$
\left\{h\left(\frac{|y|}{t}\right)^{k}\left(\frac{t}{|y|}\right)^{\sigma}\right\}^{\xi}
$$

is bounded and linear from $L_{*}^{\eta \mid \xi}\left(\mathbf{R}^{+}\right)$into $L_{*}^{\eta \mid \xi ; n-s}\left(\mathbf{R}^{n}\right)$, where $0<\sigma<k$, $\xi \leqq \eta$. Thus (3.5) is a consequence of (3.4) and Jessen's inequality (generalized Minkowski inequality). This completes the lemma.

Next consider the analogous inequalities for functions belonging to a Besov space:

Lemma 3.2. Let $\Omega$ be an open set in $\mathbf{R}^{n}$, and assume that $\Psi(x)$ is bounded and continuous. For every function $f \in B_{p, \xi}^{\tau, j}(\Omega), 0<\tau<j, 0<(2 j$ $-1) t<T_{0}$, we define

$$
\begin{aligned}
U_{j}(t, x)=\int_{B} \int_{(2 j-1) B} \mid \sum_{\nu=0}^{j}(-1)^{\nu}\left(\begin{array}{l}
j \\
\nu
\end{array}\right) & f\left(x+\mathrm{t} \frac{(j-\nu) z+\nu w}{j}\right. \\
& +t \Psi(x)) \mid d z d w .
\end{aligned}
$$

Let $1 \leqq p \leqq q \leqq \infty, 1 \leqq \xi, \eta \leqq \infty, 0<\sigma<k$. Set $\lambda=n / p-s / q$. Then:

(i) For any $f \in B_{p, \xi}^{r, j}(\Omega)$ and $0<(2 j-1) t<T_{0}$

$$
\begin{aligned}
\left\|U_{j}(t, x)\right\|_{L^{q, n-s}(\Omega)} & \leqq C_{1} t^{-\lambda} \int_{2 B} F_{j}(t z) d z, \\
& \leqq C t^{\tau-\lambda}|f|_{B_{p, \xi}^{\tau, j,}(\Omega)},
\end{aligned}
$$

where $C$ is a constant independent of $f$ and $t$, and

$$
F_{j}(y)=\left\|\sum_{\nu=0}^{j}(-1)^{\nu}\left(\begin{array}{l}
j \\
\nu
\end{array}\right) f(x+\nu y)\right\|_{L^{p}\left(\Omega_{j, y}\right)}, \Omega_{j, y}=\bigcap_{\nu=0}^{j}(\Omega-\nu y) .
$$


(ii) Assume that $l+\tau-\lambda \geqq 0$. For every $f \in B_{p, \xi}^{\tau, j}(\Omega)$ and $0<$ $(2 j-1) T<T_{0}$

$$
\left\{\int_{0}^{T}\left[\mid i U_{j}(t, x) t^{l} \|_{L^{q ; n-s}(\Omega)}\right]^{\eta} \frac{d t}{t}\right\}^{1 / \eta} \leqq C T^{l+\tau-\lambda}|f|_{B_{p, \xi}^{\tau, j},{ }_{\xi}(\Omega)},
$$

where $C$ is a constant independent of $f$ and $T$, if $l-\lambda+\tau>0$ or if $\xi \leqq \eta$. And

$$
\left\|\left\{\int_{0}^{T}\left(U_{j}(t, x) t^{l}\right)^{r} \frac{d t}{t}\right\}^{1 / r}\right\|_{L^{q, n-s}(\Omega)} \leqq C T^{l+\tau-\lambda}|f|_{B p_{p}^{r, j},{ }_{\xi}(\Omega)}
$$

where $C$ is a constant independent of $f$ and $T$, if $r \leqq q$ and if either one of the conditions (a) $l+\tau>\lambda$, (b) $\xi \leqq q<\infty, p<q, r<q$, or (c) $\xi \leqq r$ is satisfied.

(iii) For any $f \in B_{p, \xi}^{\tau, j}(\Omega)$ and $0<(2 j-1) T<T_{0}$

$$
\begin{gathered}
\left\|\left[\int_{0}^{T}\left\{\left\|U_{j}(t, x)\right\|_{L^{q, n-s}(\Omega)} h\left(\frac{|y|}{t}\right)^{k}|y|^{-\sigma} t^{l}\right\}^{r} \frac{d t}{t}\right]^{1 / r}\right\|_{L_{k}^{\eta \cdot n-s}\left(\mathbf{R}^{n}\right)} \\
\leqq C T^{l+\tau-\sigma-\lambda}|f|_{B_{p}^{\tau, j}, \xi(\Omega)},
\end{gathered}
$$

where $C$ is a constant independent of $f$ and $T$, if $r \leqq \eta, l+\tau \geqq \sigma+\lambda, \xi \leqq \eta$ or if $l+\tau>\sigma+\lambda$.

Proof. Denoting the characteristic function of $\Omega$ by $e(x)$, we find that the function $U_{j}(t, x)$ is not greater than

$$
\begin{gathered}
j^{n} \int_{b B} \int_{2 B}\left\{\prod_{\nu=0}^{j} e(x+t z+t \nu w)\right\}\left|\sum_{\nu=0}^{j}\left(\begin{array}{l}
j \\
\nu
\end{array}\right)(-1)^{\nu} f(x+t z+t \nu w)\right| d z d w \\
=j^{n} \int_{2 B} d w \int_{b B} G_{j, t w}(x+t z) d z
\end{gathered}
$$

where

$$
G_{j, w}(u)=\left[\prod_{\nu=0}^{j} e(u+\nu w)\right]\left|\sum_{\nu=0}^{j}(-1)^{\nu}\left(\begin{array}{l}
j \\
\nu
\end{array}\right) f(u+\nu w)\right|,
$$

and hence we get

$$
\left\|U_{j}(t, x)\right\|_{L^{q ; n-s}(\Omega)} \leqq j^{n} \int_{2 B} d w\left\|\int_{b B} G_{j, t w}(x+t z) d z\right\|_{L^{q: n-s}(\Omega)}
$$


From (3.2) it follows that

$$
\begin{aligned}
\left\|\int_{b B} G_{j, t w}(x+t z) d z\right\|_{L^{q: n-s}(\Omega)} & \leqq C_{1} t^{-\lambda}\left\|G_{j, t w}(u)\right\|_{L^{p}(\Omega)} \\
& =C_{1} t^{-\lambda} F_{j}(t w) .
\end{aligned}
$$

Therefore we obtain the first inequality in (3.8), which implies the second inequality in virtue of Hölder's inequality.

(ii) Proof of (3.9). We may consider only the case where $\xi \leqq \eta$ and $l+\tau=\lambda$. From (3.8) it follows that

$$
\begin{aligned}
\left\|U_{j}(t, x) t^{l}\right\|_{L^{q, n-s}(\Omega)} & \leqq C t^{-\tau} \int_{2 B} F_{j}(t z) d z, \\
& =C \int_{2 t B}(|u| / t)^{n+\tau}|u|^{-\tau} F_{j}(u)|u|^{-n} d u .
\end{aligned}
$$

Applying Lemma 2.5 to the last integral transformation, we obtain (3.9).

Assume that $p<q<\infty$ and $l+\tau=\lambda$. Note the

$$
g(x)=\int_{0}^{T}\left\{U_{j}(t, x) t^{l}\right\}^{r} \frac{d t}{t}=\int_{0}^{\infty} u(t, x) t^{-1} d t
$$

where $u(t, x)$ is the function which is equal to $\left\{t^{l} U_{j}(t, x)\right\}^{r}$ for $0<t \leqq T$ and vanishes otherwise. Set $\theta=1-r \rho / q$ and $\kappa=-s / \rho$, where $r \rho=\max$ $(p, r)$. From (3.9) we obtain

$$
\left\|\left[\left\|t^{\kappa(\theta-1)} u(t, x)\right\|_{L^{\infty}(\Omega)}\right]\right\|_{L_{i}^{\zeta}\left(\mathbf{R}^{+}\right)} \leqq\left\{C|f|_{B_{p, \xi}^{r, j}(\Omega)}^{r, r}\right\}^{r},
$$

where $r \zeta=\max (\xi, r)$, and

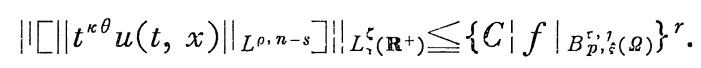

Hence $g \in\left(L^{\rho ; n-s)}(\Omega), L^{\infty}(\Omega)\right)_{\theta, \zeta} C L^{(q \mid r, \zeta) ; n-s}(\Omega)$, in view of Lemma 2.4, and we can conclude that

$$
\|\left[\int_{0}^{T}\left\{U_{j}(t, x) t^{l,\}^{r}} \frac{d t}{t}\right]^{1 / r} \|_{L^{(q, \xi), n-s(\Omega)}} \leqq C T^{l+\tau-\lambda}|f|_{B_{p, \xi \xi}^{\tau, \gamma}(\Omega)} .\right.
$$

If $\xi \leqq q$, this implies $(3.10)$, since $L^{(q, \xi)} \subset L^{q}$.

When $\xi \leqq r,(3.10)$ is verified by (3.9) and Jessen's inequality. 
(iii) We may assume that $l+\tau=\sigma+\lambda, r \leqq \eta$. From the fact stated in the proof of Lemma 3.1 (iii) it follows that

$$
\begin{gathered}
\left\|\left[\int_{0}^{T}\left\{\left\|U_{j}(t, x)\right\|_{L^{q ; n-s}(\Omega)} h\left(\frac{|y|}{t}\right)^{k}|y|^{-\sigma} t^{l}\right\}^{r} \frac{d t}{t}\right]^{1 / r}\right\|_{L_{*}^{\eta, n-s}\left(\mathbf{R}^{n}\right)} \\
\leqq C\left[\int_{0}^{T}\left\|U_{j}(t, x) t^{l-\sigma}\right\|_{L^{q ; n-s}(\Omega)}^{\eta} \frac{d t}{t}\right]^{1 / \eta} \cdot
\end{gathered}
$$

This combined with (3.9) gives (3.11), and the lemma is established.

By $\mathscr{B}^{j-}(\Omega)$ we denote the space of all functions which have bounded and uniformly Lipschitz continuous derivatives up to order $j-1$.

From the preceding lemmas we obtain

Lemma 3.3. Let $\Omega$ be an open set in $\mathbf{R}^{n}$, and let $K(x, z) \in \mathscr{B}^{i-}\left(\mathbf{R}^{n}\right.$ $\left.\times \mathbf{R}^{n}\right)$, supp $K \subset \mathbf{R}^{n} \times B$. Assume that $1 \leqq p \leqq q \leqq \infty, 1 \leqq \xi, \eta \leqq \infty, 0<\tau<j$, $0<\sigma<i$. Set $\lambda=n / p-s / q$.

For any function $f \in C^{1}(\Omega)$ and $0<t<T_{0}$ we define

$$
V(t, x)=\int K(t, x) f(x+t z+t \Psi(x)) d z .
$$

Then:

(A) (i) For any $f \in L^{p}(\Omega) \cap C^{1}(\Omega)$ and $0<t<T_{0}$

$$
\begin{aligned}
& \|V(t, x)\|_{L^{q ; n-s}(\Omega)} \leqq C t^{-\lambda}\|f\|_{L^{p}(\Omega)}, \\
& |V(t, x)|_{B_{q ; \eta}^{\sigma, i n-s}(\Omega)} \leqq C\left(t^{-\lambda-\sigma}+t^{-\lambda}\right)\|f\|_{L^{p}(\Omega)},
\end{aligned}
$$

where $C$ is a constant independent of $f$ and $t$.

(ii) There is a constant $C$ such that for any $f \in L^{p}(\Omega) \cap C^{1}(\Omega)$ and $0<T<T_{0}$

$$
\left\|\int_{0}^{T} V(t, x) t^{l-1} d t\right\|_{L^{q, n-s}(\Omega)} \leqq C T^{l-\lambda}\|f\|_{L^{p}(\Omega)},
$$

if either one of the condition (a) $l>\lambda$, or (b) $l \geqq \lambda, 1<p<q<\infty$ is satisfied.

(iii) There is a constant $C$ such that for any $f \in L^{p}(\Omega) \cap C^{1}(\Omega)$ and $0<T<T_{0}$ 


$$
\left|\int_{0}^{T} V(t, x) t^{l-1} d t\right|_{\substack{B_{q, \eta}^{\sigma} ; n-s(\Omega) \\ f(i)}} \leqq C\left(1+T^{\sigma}\right) T^{l-\sigma-\lambda} \| f_{\|^{p}(\Omega)},
$$

if $\sigma+\lambda \leqq l$, and if either one of the conditons (a) $\sigma+\lambda<l$, (b) $1<p<q$ $\leqq \infty, p \leqq \eta$, (c) $1<p \leqq \eta \leqq \infty, s<n$, or (d) $\eta=\infty$ is satisfied.

(B) Under the additional assumption that

$$
K(x, z)=\sum_{|\alpha|=j-1} D_{z}^{\alpha} H_{\alpha}(x, z),
$$

where $D_{z}^{\beta} H_{\alpha}(x, z) \in \mathscr{B}^{i-}\left(\mathbf{R}^{n} \times \mathbf{R}^{n}\right)$ for any $\alpha$ with $|\alpha|=j-1$ and any $\beta$ with $|\beta| \leqq j-1$, and that

$$
\text { supp } H_{\alpha} \subset \mathbf{R}^{n} \times B, \int H_{\alpha}(x, z) d z=0
$$

for any $|\alpha|=j-1$, there is a constant $C$ such that for every $f \in B_{p, \xi}^{r, j}(\Omega)$ $\cap C^{j}(\Omega)$ and $0<t<T$ (or $\left.0<T<T_{0}\right)$

(i)

$$
\begin{aligned}
& \|V(t, x)\|_{L^{q ; n-s}(\Omega)} \leqq C t^{\tau-\lambda}|f|_{B_{p, \xi}^{\tau, j}(\Omega)}, \\
& |V(t, x)|_{B_{q, \eta, n-s}^{\sigma, i}(\Omega)} \leqq C\left(t^{\tau-\sigma-\lambda}+t^{\tau-\lambda}\right)|f|_{B_{p, \xi}^{r, j}(\Omega)}
\end{aligned}
$$

(ii)

$$
\left\|\int_{0}^{T} V(t, x) t^{l-1} d t\right\|_{L^{q ; n-s}(\Omega)} \leqq C T^{l+\tau-\lambda}|f|_{B_{p ; \xi}^{\tau, j}, j(\Omega)}
$$

if $l+\tau \geqq \lambda$ and if either one of the conditions (a) $l+\tau>\lambda$, (b) $\xi \leqq q<\infty$, $p<q$, or (c) $\xi=1$ is satisfied; and

(iii)

$$
\left|\int_{0}^{T} V(t, x) t^{l-1} d t\right|_{B_{q, \eta n-s}^{\sigma, i}(\Omega)} \leqq C\left(1+T^{\sigma}\right) T^{l+\tau-\sigma-\lambda}|f|_{B_{p, \xi}^{\mathrm{r}, j},(\Omega)} .
$$

if either (a) $l+\tau-\sigma-\lambda>0$, or (b) $l+\tau-\sigma-\lambda \geqq 0, \xi \leqq \eta$ is satisfied.

Proof. Note that

$$
\left|\sum_{\nu=0}^{i}(-1)^{\nu}\left(\begin{array}{l}
i \\
\nu
\end{array}\right) V(t, x+\nu y)\right| \leqq C_{1}\left\{h\left(\frac{|y|}{t}\right)^{i}+h(|y|)^{i}\right\} \sum_{\nu=0}^{i} U_{j}(t, x+\nu y)
$$

(see proof of [7] Lemma 2.6), then the assertions follow from Lemma 3.1 and Lemma 3.2 . 
Proof of Theorem 1 and Theorem 2. To prove Theorem 1 it is enough to show that the inequalities (1.5) and (1.6) hold for every $f \in$ $W_{p}^{m}(\Omega) \cap C^{\infty}(\Omega)$. From Lemma 2.1 in $[5]$ it follows that for any $f \in$ $C^{m}(\Omega)$ and $|\beta|<m$

$$
\begin{gathered}
D^{\beta} f(x)=\sum_{|\alpha|=m, \alpha \geqq \beta} \int_{0}^{T} t^{m-|\beta|-1} d t \int \omega_{\alpha-\beta}(x, z) D^{\alpha} f(x+t z+t \Psi(x)) d z \\
+T^{-|\beta|} \int \omega_{m, \beta}(x, z) f(x+T z+T \Psi(x)) d z,
\end{gathered}
$$

which gives the desired inequalities with the aid of Lemma 3.3 (A).

In the same way Theorem 2 is verified by Lemma 3.3 (B) and the integral representation $\left(2.1^{\prime}\right)$ in $[7]$.

\section{Appendix A}

Let $\Phi(t), t \in \mathbb{R}^{+}$, be a convex non-decreasing, positive function such that $\Phi(t) / t \rightarrow 0$ as $t \rightarrow 0$ and that $\Phi(t) / t \rightarrow+\infty$ as $t \rightarrow+\infty$. By $L^{\Phi}(\Omega)$ we write the space of measurable functions $f(x)$ such that there is $\lambda>0$ with

$$
\int \Phi(\lambda|f(x)|) d x \leqq 1
$$

With respect to the norm

$$
\|f\|_{\emptyset}=\inf \left\{\lambda^{-1} ; \int \Phi(\lambda|f(x)|) d x \leqq 1\right\}
$$

$L^{\Phi}(\Omega)$ is a Banach space, and is called a Orlicz space.

As a supplement to the imbedding theorem, we have the following theorem concerning the limiting case.

Theorem A. The imbedding operator

$$
B_{p, \infty}^{\tau}(\Omega) \rightarrow L^{\Phi_{p}}(\Omega)
$$

exists if $\tau=n / p$, where

$$
\Phi_{p}(t)=\sum_{j \geqq p} \frac{t^{j}}{j !}
$$

Proof. Let $q \geqq p$. It follows from our proof of Theorem 2 that 


$$
\|f\|_{L^{q}(\Omega)} \leqq C_{1} q T^{\tau-\lambda}|f|_{B_{p, \infty}^{\tau}(\Omega)}+C_{2} T^{-\lambda}\|f\|_{L^{p}(\Omega)}
$$

where $\lambda=n / p-n / q, 0<T<T_{0} / 3$, and where $C_{1}, C_{2}$ are constant independent of $f, T$, and $q$. Take a fixed $T_{1}<T_{0} / 3$, and put

$$
\begin{gathered}
A=C_{1}|f|_{B_{p, \infty}^{\tau}(\Omega)}, \quad B=C_{2} \mid i f \|_{L^{p}(\Omega)}, \\
k=\max \left(p, B A^{-1} T_{1}^{-n / p}\right) .
\end{gathered}
$$

Then we have,

$$
\begin{gathered}
\|f\|_{L^{q}(\Omega)} \leqq 2 B^{p / q}(q A)^{1-p / q} \quad \text { if } q \geqq k, \\
\left(\text { take } T=(B / q A)^{p / n}\right) \\
\|f\|_{L^{q}(\Omega) \leqq} T_{1}^{n / q} B T_{1}^{-n / p} \quad \text { if } p \leqq q<k, \\
\text { (take } \left.T=T_{1}\right) .
\end{gathered}
$$

Consequently we obtain

$$
\begin{aligned}
& \sum_{j \geqq p} \frac{\lambda^{j}}{j !} \int|f(x)|^{j} d x \leqq T_{1}^{n} \sum_{k>j \geqq p} \frac{(2 \lambda B)^{j}}{j !} T_{1}^{-n j \mid p} \\
& +\left(\frac{B}{A}\right)^{p} \sum_{j \geqq k} \frac{(2 \lambda A)^{j}}{j !} j^{j-p} \\
& \leqq T_{1}^{n} \sum_{k>j \geqq p}\left(2 \lambda B T_{1}^{-n / p}\right)^{j}+\left(\frac{B}{A}\right)^{p} \sum_{j \geqq k}(2 e \lambda A)^{j} .
\end{aligned}
$$

Therefore

$$
\int \Phi_{p}(\lambda|f(x)|) d x \leqq 1
$$

if

$$
4 e A \lambda \leqq 1 \text { and } 4\left(e+T_{1}^{-n / p}\right) \lambda B
$$

This implies that

$$
\|f\|_{\Phi_{p}} \leqq 4 e A+4\left(e+T_{1}^{-n / p}\right) B
$$


which is the desired inequality.

\section{Appendix B}

In this appendix we prove a theorem, half of which is contained in Theorem 1.4 in $[7]$.

Theorem B. Let $\mu_{0}, \mu_{1}>0,1 \leqq p_{0}, p_{1}, q_{0}, q_{1} \leqq \infty$ and let $0<\theta<1$. Set

$$
\begin{aligned}
& \frac{1}{p}=\frac{1-\theta}{p_{0}}+\frac{\theta}{p_{1}}, \frac{1}{q}=\frac{1-\theta}{q_{0}}+\frac{\theta}{q_{1}}, \mu=(1-\theta) \mu_{0}+\theta \mu_{1}, \\
& \mathscr{B}_{0}=B_{p_{0}, q_{0}}^{\mu_{0}}(\Omega), \mathscr{B}_{1}=B_{p_{1}, q_{1}}^{\mu_{1}}(\Omega) .
\end{aligned}
$$

Then, (i) if $r \geqq p$ and $r \geqq q$,

$$
\left(\mathscr{B}_{0}, \mathscr{B}_{1}\right)_{\theta, r} \supset B_{p, q}^{\mu}(\Omega) .
$$

(ii) If $r \leqq p$ and $r \leqq q$,

$$
\left(\mathscr{B}_{0}, \mathscr{B}_{1}\right)_{\theta, r} \subset B_{p, q}^{\mu}(\Omega) \text {. }
$$

All injections above are continuous.

The theorem is proved in virtue of the following lemmas.

\section{Lemma B.1.}

(a) $W_{p}^{m}(\Omega) \subset\left(B_{p_{0}, \infty}^{m}(\Omega), B_{p_{1} \infty}^{m}(\Omega)\right)_{\theta, q}$, if $q \geqq p$.

(b) $W_{p}^{m}(\Omega) \subset\left[B_{p_{0}, \infty}^{m}(\Omega), B_{p_{1}, \infty}^{m}(\Omega)\right]_{\theta}$.

Proof. Set $k=n^{m}+1$. Define the linear operator $L:\left\{L^{p}(\Omega)\right\}^{k} \rightarrow B_{p, \infty}^{m}(\Omega)$ by

$$
\begin{aligned}
L\left\{f_{\alpha}\right\}= & \sum_{|\alpha|=m}(-1)^{m} m \int_{0}^{T} t^{m-1} d t \int K_{\alpha}(x, z) f_{\alpha}(x+t z+t \Psi(x)) d z \\
& +\int \omega_{m+2}(x, z) f_{0}(x+T z+T \Psi(x)) d z,
\end{aligned}
$$

where

$$
K_{\alpha}(x, z)=\frac{2}{m(m+1)} \sum_{|\gamma|=2}\left(\begin{array}{c}
\alpha+\gamma \\
\gamma
\end{array}\right) D_{z}^{\gamma} \omega_{\alpha+\gamma}(x, z)
$$


It follows from Lemma 3.3 that $L$ is bounded. Also with the aid of Lemma 2.1 corollary in $[7]$ we have

$$
L\left(\left\{f^{(\alpha)}\right\}_{|\alpha|=m \text { or } 0}\right)=f
$$

for every $f \in W_{p}^{m}(\Omega) \cap C^{\infty}(\Omega)$.

Applying the interpolation theorem, we see

$$
L:\left(\left\{L^{p_{0}}(\Omega)\right\}^{k},\left\{L^{p_{1}}(\Omega)\right\}^{k}\right)_{\theta, q} \rightarrow\left(B_{p_{0}, \infty}^{m}(\Omega), B_{p_{1}, \infty}^{m}(\Omega)\right)_{\theta, q}
$$

is bounded. Combining this with

$$
\left(L^{p_{0}}, L^{p_{1}}\right)_{\theta, q}=L^{(p, q)} \supset L^{p},
$$

and that

$$
\left\{f^{(\alpha)}\right\}_{|\alpha|=m, 0} \in\left(L^{p}(\Omega)\right)^{k}
$$

for every $f \in W_{p}^{m}(\Omega)$, we have part (a). Part (b) can be proved similarly.

Let $(M, \mu)$ be a measure space, and let $\rho(x)$ be a non-negative measurable function. By $L^{p, \rho}(M ; X)$ we denote the Banach space of functions $f$ such that $\rho f \in L^{p}(M ; X)$.

Lemma B.2. Let $X_{0}, X_{1}$ be two Banach spaces contained in a Hausdorff linear topological space, and let $1 \leqq q_{0}, q_{1}, p \leqq \infty, 0<\theta<1$. Set

$$
\begin{aligned}
& \frac{1}{q}=\frac{1-\theta}{q_{0}}+\frac{\theta}{q_{1}}, X_{\theta, p}=\left(X_{0}, X_{1}\right)_{\theta, p}, \\
& \rho(x)=\rho_{0}(x)^{1-\theta} \rho_{1}(x)^{\theta}
\end{aligned}
$$

where $\rho_{0}, \rho_{1}$ be non-negative measurable functions,

(i) If $p \leqq q$, then

$$
\left(L^{q_{0}, \rho_{0}}\left(M ; X_{0}\right), L^{q_{1}, \rho_{1}}\left(M ; X_{1}\right)\right)_{\theta, p} \subset L^{q, \rho}\left(M ; X_{\theta, p}\right)
$$

with continuous injection.

(ii) If $(M, \mu)$ is $\sigma$-finite and if $q \leqq p, q<+\infty$, then

$$
L^{q, \rho}\left(M ; X_{\theta, p}\right) \subset\left(L^{q_{0}, \rho_{0}}\left(M ; X_{0}\right), L^{q_{1}, \rho_{1}}\left(M ; X_{1}\right)\right)_{\theta, p}
$$

with continuous injection. 
Proof. First we note that

$$
\left(Y_{0}, Y_{1}\right)_{\theta, p}=S\left(p_{0}, \theta, Y_{0} ; p_{1}, \theta-1, Y_{1}\right)
$$

where $1 / p=(1-\theta) / p_{0}+\theta / p_{1}$ (Peetre [12]).

(i) Since $p \leqq q$, we can choose $p_{0} \leqq q_{0}, p_{1} \leqq q_{1}$. Let

$$
f(x)=\int_{0}^{\infty} u(t, x) \frac{d t}{t}
$$

where

$$
\begin{gathered}
u(t, x) \in L_{*}^{p_{0}, \theta}\left(\mathbb{R}^{+} ; L^{q_{0}, \rho_{0}}\left(M ; X_{0}\right)\right) \cap L_{*}^{p_{1}, \theta-1}\left(\mathbb{R}^{+} ; L^{q_{1}, \rho_{1}}\left(M ; X_{1}\right)\right) \\
\left(L_{*}^{p, \alpha}\left(\mathbb{R}^{+} ; X\right)=L^{p, t^{\alpha}}\left(\mathbb{R}^{+}, t^{-1} d t ; X\right)\right) .
\end{gathered}
$$

By a lemma due to Lions-Peetre [3]

$$
\|f\|_{X_{\theta, p}} \leqq\left\|t^{\theta} u(t, x)\right\|_{L_{*}^{p^{0}}\left(\mathbf{R}^{+} ; X_{0}\right)}^{1}\left\|t^{\theta-1} u(t, x)\right\|_{L_{*}^{p_{1}}\left(\mathbf{R}^{+} ; X_{1}\right)} \cdot
$$

So that, in view of Hölder's inequality and Jessen's inequality,

$$
\begin{aligned}
& \|\rho(x) f(x)\|_{L^{q}\left(M ; X_{\theta, p}\right)} \\
& \leqq\left\|\rho_{0}(x) t^{\theta} u(t, x)\right\|_{L_{*}^{q}\left(M ; L_{*}^{q_{0}}\left(\mathbf{R}^{+} ; X_{0}\right)\right)}^{1} \\
& \times\left\|\rho_{1}(x) t^{\theta-1} u(t, x)\right\|_{L^{p_{1}}\left(M ; L_{*}^{p_{1}}\left(\mathbf{R}^{+} ; X_{1}\right)\right)}
\end{aligned}
$$

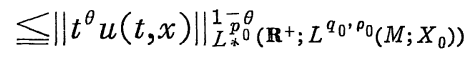

$$
\begin{aligned}
& \times\left\|t^{\theta-1} u(t, x)\right\|_{L_{*}^{p_{1}}\left(\mathbf{R}^{+} ; L^{q_{1}, \rho_{1}}\left(M ; X_{1}\right)\right)},
\end{aligned}
$$

which implies part (i).

Proof of part (ii). It is sufficient to show that for any simple $X_{\theta, p^{-}}$ valued function $f$ there exists $u(t, x)$ such that

$$
\begin{aligned}
& \left\|t^{\theta} \rho_{0}(x) u(t, x)\right\|_{L_{*}^{p_{0}}\left(\mathbf{R}^{+} ; L^{q_{0}}\left(M ; X_{0}\right)\right.}, \\
& \left\|t^{\theta-1} \rho_{1}(x) u(t, x)\right\|_{L_{*}^{p_{1}}\left(\mathbf{R}^{+} ; L^{q_{1}}\left(M ; X_{1}\right) \leqq C\|f\|_{L^{q, \rho}\left(M ; X_{\theta, p}\right),}\right.} \\
& f(x)=\int_{0}^{\infty} u(t, x) \frac{d t}{t} .
\end{aligned}
$$


Let

$$
f(x)=\sum_{\nu} a_{\nu} \psi_{\nu}(x)
$$

where $a_{\nu} \in X_{\theta, p}$, and $\left\{\psi_{\nu}\right\}$ are characteristic functions of measurable, disjoint sets. By definition for any $c>1$ we can find $u_{\nu}(t)$ such that

$$
a_{\nu}=\int_{0}^{\infty} u_{\nu}(t) \frac{d t}{t}
$$

with

$$
\left\|t^{\theta} u_{\nu}\right\|_{L_{*}^{p_{0}\left(\mathbf{R}^{+} ; X_{0}\right)}}, \quad\left\|t^{\theta-1} u_{\nu}\right\|_{L_{*}^{p_{1}\left(\mathbf{R}^{+} ; X_{1}\right)} \leqq c}\left\|a_{\nu}\right\|_{X_{\theta, p}}
$$

Then

$$
u(t, x)=\sum_{\nu} u_{\nu}(\varphi(x) t) \psi_{\nu}(x)
$$

where $\varphi(x)=\left\{\|f(x)\|_{X_{\theta, p}} \sigma^{-1}\right\}^{\lambda} \rho_{0}(x)^{q / q_{1}} \rho_{1}(x)^{-q / q_{0}}$

$$
\sigma=\|f\|_{L^{q \cdot \rho}\left(M ; X_{0, p)}\right)}, \lambda=\frac{q_{0}-q}{q_{0} \theta}
$$

has the required property.

Lemma B.3. (a generalization of the commutativity theorem due to Grisvard). Let $X_{0}, X_{1}, Y_{0}, Y_{1} \subset \mathscr{X}$ be Banach spaces.

(i) Assume that there exists a linear operator $E$ from $\mathscr{X}$ into $L_{\text {loc }}^{1}\left(\mathbb{R}^{+} ; \mathscr{X}\right)$ such that $E$ is continuous from $S_{i}$ into $W_{i},(i=0,1)$ and $P E f=f$ for every $f \in S_{0}+S_{1}$, where

$$
\begin{aligned}
& S_{i}=\left(X_{i}, Y_{i}\right)_{\sigma_{i}, q_{\imath}}, \\
& W_{i}=L_{*}^{q_{i}, \lambda_{i} \sigma_{i}}\left(\mathbb{R}^{+} ; X_{i}\right) \cap L_{*}^{q_{*}, \lambda_{\imath}\left(\sigma_{2}-1\right)}\left(\mathbb{R}^{+} ; Y_{i}\right), \quad(i=0,1) \\
& P u=\int_{0}^{\infty} u(t) \frac{d t}{t} .
\end{aligned}
$$

Set $X_{\theta, p}=\left(X_{0}, X_{1}\right)_{\theta, p}, \quad Y_{\theta, p}=\left(Y_{0}, Y_{1}\right)_{\theta, p} . \quad$ If $p \leqq q$, then

$$
\left(S_{0}, S_{1}\right)_{\theta, p} \subset\left(X_{\theta, p}, Y_{\theta, p}\right)_{\sigma, q}=S_{\theta},
$$


where $\lambda=(1-\theta) \lambda_{0}+\theta \lambda_{1}, \quad \lambda \sigma=(1-\theta) \lambda_{0} \sigma_{0}+\theta \lambda_{1} \sigma_{1}$.

(ii) Assume that there exists a linear operator $J$ from $\mathscr{L}_{0}+\mathscr{L}_{1}$ into $\mathscr{X}$ such that $J$ is continuous from $\mathscr{L}_{i}$ into $S_{i}(i=0,1)$, and $J u=f$ if $u(t)$ $=f$ a.e.t. Here

$$
\mathscr{L}_{i}=L_{*}^{q_{i}, \lambda_{i} \sigma_{i}}\left(\mathbf{R}^{+} ; X_{i}\right)+L_{*}^{q_{i}, \lambda_{i}\left(\sigma_{i}-1\right)}\left(\mathbf{R}^{+} ; Y_{i}\right)
$$

If $p \geqq q$, then $S_{\theta} \subset\left(S_{0}, S_{1}\right)_{\theta, p}$.

Proof. (i) From the interpolation theorem it follows that $E$ is continuous from $\left(S_{0}, S_{1}\right)_{\theta, p}$ into $\left(W_{0}, W_{1}\right)_{\theta, p}$. In view of Lemma B.2.(i) we see

$$
\left(W_{0}, W_{1}\right)_{\theta, p} \subset L_{*}^{q, \lambda \sigma}\left(\mathbf{R}^{+} ; X_{\theta, p}\right) \cap L_{*}^{q, \lambda(\sigma-1)}\left(\mathbf{R}^{+} ; Y_{\theta, p}\right)
$$

Therefore, $P E$ is a continuous injection from $\left(S_{0}, S_{1}\right)_{\theta, p}$ into $S_{\theta}$.

(ii) By interpolation we see that $J$ maps continuously $\left(\mathscr{L}_{0}, \mathscr{L}_{1}\right)_{\theta, p}$ into $\left(S_{0}, S_{1}\right)_{\theta, p}$. It follows from Lemma B.2. (ii) that $J$ is a bounded linear operator from

$$
L_{*}^{q, \lambda \sigma}\left(\mathbf{R}^{+} ; X_{\theta, p}\right)+L_{*}^{q, \lambda(\sigma-1)}\left(\mathbf{R}^{+} ; Y_{\theta, p}\right)
$$

into $\left(S_{0}, S_{1}\right)_{\theta, p}$, so that we have (ii). This completes the lemma.

Proof of the theorem. Part (i). Combining Lemma B.1, Lemma B.3.(ii) and Lemma 4.3 in [7] (this lemma is still valid if we replace $W_{p}^{m}$ by $B_{p, \infty}^{m}$ ), we have

$$
\begin{aligned}
& \left(\mathscr{B}_{0}, \mathscr{B}_{1}\right)_{\theta, r} \\
& \supset\left(\left(L^{p_{0}}, L^{p_{1}}\right)_{\theta, r},\left(B_{p_{0}, \infty}^{m}, B_{p_{1}, \infty}^{m}\right)_{\theta, r}\right)_{\sigma, q} \\
& \supset\left(L^{p}, W_{p}^{m}\right)_{\sigma, q}=B_{p, q}^{\mu},
\end{aligned}
$$

where $\mu_{i}=\sigma_{i} m(i=0,1), \mu=\sigma m$.

Part (ii).

In view of Lemma B.3.(i), Lemma 4.2 in [7] and the fact that

$$
\left(W_{p_{0}}^{m}, W_{p_{1}}^{m}\right)_{\theta, r} \subset W_{p}^{m}
$$


(apply the interpolation theorem to the operator $f \rightarrow D^{\alpha} f$ ), we see that

$$
\begin{gathered}
\left(\mathscr{B}_{0}, \mathscr{B}_{1}\right)_{\theta, r} \subset\left(\left(L^{p_{0}}, L^{p_{1}}\right)_{\theta, r},\left(W_{p_{0},}^{m} W_{p_{1}}^{m}\right)_{\theta, r}\right)_{\sigma, q} \\
C\left(L^{p}, W_{p}^{m}\right)_{\sigma, q}=B_{p, q}^{\mu} .
\end{gathered}
$$

\section{References}

[1] Besov, O. V., Investigation of a family of function spaces in connection with theorems of imbedding and extension, Trudy Mat. Inst. Steklov 60 (1961), 42-81. (Russian) = A.M.S. Transl. (2) 40 (1964), 85-126.

[2] Il'in, V. P., The properties of some classes of differentiable functions of several variables in an $n$-dimensional region, Trudy Mat. Inst. Steklov 66 (1962) 227-363 (Russian) = A.M.S. Transl. (2) 81 (1969), 91-256.

[3] Lions, J. L. and J. Peetre, Sur une classe d'espaces d'interpolation, Publ. Math. I.H.E.S. 19 (1964), 5-68.

[4] Lorents, G. G., Some new functional spaces, Ann. of Math. (2) 51 (1950), 37-55.

[5] Muramatu, T., On imbedding theorems for Sobolev spaces and some of their generalization, Publ. Res. Inst. Math. Sci. Kyoto Univ. 3 (1968), 393-416.

[6] Products of fractional powers of operators, J. Fac. Sci. Univ. Tokyo, Sect. I A, 17 (1970), 581-590.

[7] - T., On Besov spaces of functions defined in general regions, to appear.

[8] Nikol'skii, S. M., On imbedding, continuation and approximation theorems for differentiable functions of several variables, Uspehi. Mat. Nauk 16, No. 5 (1961), 63-114 (Russian)=Russian Math. Surveys 16, No. 5 (1961), 55-104.

[9] Oklander, E. T., $L_{p q}$ interpolators and the theorem of Marcinkiewicz, Bull. Amer. Math. Soc. 72 (1966), 49-53.

[10] O'Neil, R., Convolution operators and $L(p, q)$ spaces, Duke Math. J. 30 (1963), 129-142.

[11] Peetre, J., Espaces d'interpolation et théorème de Soboleff, Ann. Inst. Fourier (Grenoble) 16 (1966), 279-317.

[12] - Sur le nombre de paramètres dans la définition de certains espaces d'interpolation, Ricerche Mat. 12 (1963), 248-261.

[13] Taibleson, M. H., On the theory of Lipschitz spaces of distribution on Euclidean n-space I, J. Math. Mech. 13 (1964), 407-479.

[14] Yoshikawa, A., An abstract formulation of Sobolev type imbedding theorems and its applications to elliptic boundary value problems, J. Fac. Sci. Univ. Tokyo, Sect. I A, 17 (1970), 543-558.

[15] - Fractional powers of operators, interpolation theory and imbedding theorems, to appear. 
